\title{
LOOP GROUPS, STRING CLASSES AND EQUIVARIANT COHOMOLOGY
}

\author{
RAYMOND F. VOZZO \\ (Received 22 July 2010; accepted 3 December 2010) \\ Communicated by S. Rosenberg \\ Dedicated to Alan Carey, on the occasion of his 60th birthday
}

\begin{abstract}
We give a classifying theory for $L G$-bundles, where $L G$ is the loop group of a compact Lie group $G$, and present a calculation for the string class of the universal $L G$-bundle. We show that this class is in fact an equivariant cohomology class and give an equivariant differential form representing it. We then use the caloron correspondence to define (higher) characteristic classes for $L G$-bundles and to prove a result for characteristic classes for based loop groups for the free loop group. These classes have a natural interpretation in equivariant cohomology and we give equivariant differential form representatives for the universal case in all odd dimensions.
\end{abstract}

2010 Mathematics subject classification: primary 55R10; secondary 55R35, 57R20, 81T30.

Keywords and phrases: loop groups, string class, caloron correspondence, equivariant cohomology.

\section{Introduction}

Let $G$ be a compact, connected, simply connected Lie group with Lie algebra $\mathfrak{g}$ and $L G$ be the space of smooth maps from the circle into $G$. Then $L G$ is a Fréchet Lie group. In this article we shall be considering characteristic classes of principal bundles whose structure group is $L G$. Recall that in [13] we constructed characteristic classes for bundles whose structure group is the based loop group, $\Omega G$, using the caloron correspondence-a correspondence between loop group bundles and certain $G$-bundles. Part of what made that construction simple was the fact that there exists a nice model for the universal bundle and hence a relatively easy classifying theory (this is summarized in Theorem 2.5). Here we would like to extend these results to the group of free loops, where the classifying theory is more complicated. Our main result, Theorem 6.5, is the analogy of Theorem 2.5 for the free loop group. We state it here for convenience.

The author acknowledges the support of the Australian Research Council.

(C) 2011 Australian Mathematical Publishing Association Inc. 1446-7887/2011 \$16.00 
TheOREM. Let $P \rightarrow M$ be an LG-bundle and $\widetilde{P} \rightarrow M \times S^{1}$ be its caloron transform. If

$$
s(P): I^{k}(\mathfrak{g}) \rightarrow H^{2 k-1}(M)
$$

is the map that gives for any $p \in I^{k}(\mathfrak{g})$ its associated string class, then the following diagram commutes.

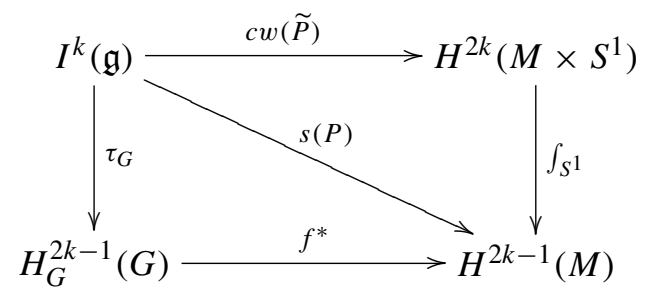

Here $f$ is the classifying map of $P$ (Section 3.2).

This article is organized as follows. In Section 2, we review the formula for the string class from [12] and the extension of this class to higher dimensions for the based loop group [13]. In Section 3, we outline the construction of the universal $L G$-bundle and give a classifying map for any $L G$-bundle. We show that characteristic classes for $L G$-bundles are given by equivariant cohomology classes on $G$. Section 4 reviews the necessary background on equivariant cohomology, in particular the Cartan model of equivariant differential forms and the Mathai-Quillen isomorphism. Sections 5 and 6 contain our main results concerning string classes: in Section 5, we calculate the universal degree-three string class, and in Section 6, we extend this to any odd degree, proving a theorem analogous to the main result in [13] for $\Omega G$ (Theorem 6.5).

\section{String classes for loop group bundles}

2.1. The string class. String structures were introduced by Killingback as the string theory analogue of spin structures [10]. Suppose that we have an $L G$-bundle $P \rightarrow M$. Since $L G$ has a central extension by the circle (see, for example, [17] for details), we can consider the problem of lifting the structure group of $P$ to the central extension $\widehat{L G}$ of $L G$. Physically, this is related to the problem of defining a Dirac-Ramond operator in string theory. Mathematically, one has an obstruction to doing this-a certain degree-three cohomology class on the base of the bundle. This class is called the string class of the bundle and we write $s(P) \in H^{3}(M)$. In [12] Murray and Stevenson give a formula for a de Rham representative of this class, as follows.

TheOREM 2.1 [12]. Let $P \rightarrow M$ be a principal LG-bundle. Let A be a connection on $P$ with curvature $F$ and let $\Phi$ be a Higgs field for $P$. Then the string class of $P$ is represented in de Rham cohomology by the form

$$
-\frac{1}{4 \pi^{2}} \int_{S^{1}}\langle\nabla \Phi, F\rangle d \theta
$$


where $\langle$,$\rangle is an invariant inner product on \mathfrak{g}$ normalized so the longest root has length equal to $\sqrt{2}$ and

$$
\nabla \Phi=d \Phi+[A, \Phi]-\frac{\partial A}{\partial \theta} .
$$

By definition, the Higgs field for $P$ in Theorem 2.1 is a map $\Phi: P \rightarrow L \mathfrak{g}$ satisfying

$$
\Phi(p \gamma)=\operatorname{ad}\left(\gamma^{-1}\right) \Phi(p)+\gamma^{-1} \partial \gamma
$$

where $\partial \gamma=\partial \gamma / \partial \theta$ is the derivative in the loop direction. Note that, like $F$, the covariant derivative $\nabla \Phi$ is equivariant for the adjoint action of $L G$ and hence the string class descends to a form on $M$. A geometric interpretation of the Higgs field is given by the caloron correspondence [12] (see also [13, 14]), which allows us to extend the definition of the string class to higher degrees. We shall discuss this correspondence in Section 2.3.

2.2. The universal string class for $\boldsymbol{\Omega} \boldsymbol{G}$-bundles. Theorem 2.1 carries over wordfor-word for the based loop group $\Omega G$. In this case there is a simple model for the universal bundle that we shall now present, and which makes it possible to easily calculate the universal string class.

Let $P G$ be the space of paths in $G$, that is smooth maps $p: \mathbb{R} \rightarrow G$ such that $p(0)=1$, the identity in $G$ and $p^{-1} \partial p$ is $2 \pi$-periodic. This is acted on by $\Omega G$ and

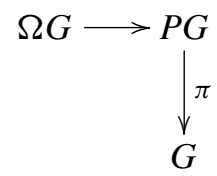

is an $\Omega G$-bundle called the path fibration, where the projection $\pi=\mathrm{ev}_{2 \pi}$ sends a path $p$ to its value at $2 \pi . P G$ is contractible and so the path fibration is a model for the universal $\Omega G$-bundle and we have $B \Omega G=G$. Given another $\Omega G$-bundle $P \rightarrow M$ we can write down a classifying map as follows [13]. Choose a Higgs field $\Phi$ for $P$. Then for each $p \in P$ we define $\operatorname{hol}_{\Phi}(p)$ to be the solution $q$ to the equation $\Phi(p)=q^{-1} \partial q$ for all $q \in P G$. (Note that $q \in P G$ implies that $q(0)=1$ which ensures that the solution is unique.) The map $\operatorname{hol}_{\Phi}$ descends to a map, also called hol $\Phi$, from $M$ to $G$. We call this map the Higgs field holonomy for $P$; it gives a classifying map for the $\Omega G$-bundle $P$.

A connection for the path fibration is given in [3]. Let $\alpha: \mathbb{R} \rightarrow \mathbb{R}$ be a smooth function such that $\alpha(t)=0$ for all $t \leq 0$ and $\alpha(t)=1$ for all $t \geq 2 \pi$. Then, at $p \in P G$,

$$
A=\Theta-\alpha \operatorname{ad}\left(p^{-1}\right) \pi^{*} \widehat{\Theta}
$$

defines a connection, where $\Theta$ is the left invariant Maurer-Cartan form on $G$ and $\widehat{\Theta}$ is the right invariant Maurer-Cartan form. The curvature of this connection is

$$
F=\frac{1}{2}\left(\alpha^{2}-\alpha\right) \operatorname{ad}\left(p^{-1}\right)\left[\pi^{*} \widehat{\Theta}, \pi^{*} \widehat{\Theta}\right] .
$$


A Higgs field for $P G$ is given by

$$
\Phi(p)=p^{-1} \partial p
$$

Its covariant derivative is

$$
\nabla \Phi=\partial \alpha \operatorname{ad}\left(p^{-1}\right) \pi^{*} \widehat{\Theta},
$$

where $\partial \alpha=\partial \alpha / \partial t$. We call these the standard connection and Higgs field for the path fibration.

Using Theorem 2.1 and the standard connection and Higgs field for the path fibration, we can calculate the universal string class for $\Omega G$-bundles $s(P G) \in H^{3}(G)$ :

$$
\begin{aligned}
s(P G) & =-\frac{1}{4 \pi^{2}} \int_{S^{1}}\left\langle\frac{1}{2}\left(\alpha^{2}-\alpha\right) \operatorname{ad}\left(p^{-1}\right)\left[\pi^{*} \widehat{\Theta}, \pi^{*} \widehat{\Theta}\right], \partial \alpha \operatorname{ad}\left(p^{-1}\right) \pi^{*} \widehat{\Theta}\right\rangle d \theta \\
& =\frac{1}{48 \pi^{2}}\langle[\Theta, \Theta], \Theta\rangle
\end{aligned}
$$

which is the generator of the degree-three cohomology of $G$.

2.3. Higher string classes for $\boldsymbol{\Omega} \boldsymbol{G}$-bundles. The material above is actually the degree-three case of a more general construction presented in [13]. In that paper we showed how to obtain higher-degree analogues of the string class for any $\Omega G$-bundle using the caloron correspondence, a correspondence between loop group bundles and certain $G$-bundles. We shall outline these results here before extending them to $L G$ bundles in Section 6. We begin with the caloron correspondence.

The caloron correspondence gives a bijection between isomorphism classes of $L G$ bundles over a manifold $M$ and isomorphism classes of $G$-bundles over $M \times S^{1}$. It was first introduced in [6] as a bijection between isomorphism classes of $G$-instantons on $\mathbb{R}^{3} \times S^{1}$ and $\Omega G$-monopoles on $\mathbb{R}^{3}$. The form we will use in this article, however, is from [12], where it was used to relate the string class of an $L G$-bundle to the Pontrjagyn class of a $G$-bundle.

Let $\widetilde{P} \rightarrow M \times S^{1}$ be a $G$-bundle and consider the $L G$-bundle $L \widetilde{P} \rightarrow L\left(M \times S^{1}\right)$. Pull this bundle back by the map $\eta: M \rightarrow L\left(M \times S^{1}\right)$ given by $\eta(m)=(\theta \mapsto(m, \theta))$. This gives an $L G$-bundle $P \rightarrow M$. Furthermore, given a connection $\widetilde{A}$ on $\widetilde{P}$, we can define a connection $A$ on $P$ by first defining a connection $L \widetilde{A}$ on $L \widetilde{P}$ given by acting pointwise, that is, $L \widetilde{A}_{\gamma}(X)(\theta)=\widetilde{A}_{\gamma(\theta)}(X(\theta))$, for any tangent vector $X$ to $\gamma \in L \widetilde{P}$, then pulling back by $\eta$, so $A=\eta^{*} L \widetilde{A}$. Note that we can also define a Higgs field as follows. Any $p \in P$ is given by a map $S^{1} \rightarrow \widetilde{P}$. We define $\Phi(p)=\widetilde{A}(\partial p)$. This gives a Higgs field for $P$.

On the other hand, given an $L G$-bundle $P \rightarrow M$, we can define a $G$-bundle $\widetilde{P}$ by $\widetilde{P}=\left(P \times G \times S^{1}\right) / L G$ where the $L G$ action is given by $(p, g, \theta) \gamma=$ $\left(p \gamma, \gamma(\theta)^{-1} g, \theta\right)$ and the $G$ action is multiplication in the second factor. In order to define a connection $\widetilde{A}$ on $\widetilde{P}$ from a connection $A$ on $P$ we also need to choose a Higgs field $\Phi$ for $P$. Then, as a form on $P \times G \times S^{1}$ which descends to the quotient,

$$
\widetilde{A}=\operatorname{ad}\left(g^{-1}\right) A(\theta)+\Theta+\operatorname{ad}\left(g^{-1}\right) \Phi d \theta .
$$

Using these constructions we can obtain the result from [12]. 
PROPOSITION 2.2 (Caloron correspondence [12]). The constructions above are inverses of one another and give a bijection between isomorphism classes of $G$ bundles with connection over $M \times S^{1}$ and isomorphism classes of LG-bundles with connection and Higgs field over $M$.

Here we will need a slight modification of this result, given in [13]. Specifically, in order to construct an $\Omega G$-bundle $P \rightarrow M$ given a $G$-bundle $\widetilde{P} \rightarrow M \times S^{1}$ we need a way of choosing a basepoint in each fibre of $P$. This is given by choosing a section of $\widetilde{P}$ over $M \times\{0\}$. Such a section is called a framing and, if it exists, $\widetilde{P}$ is called a framed $G$-bundle. A connection $A$ on $\widetilde{P}$ is called framed if it is flat with respect to the section defining the framing. We have the following proposition.

PROPOSITION 2.3 [13]. There is a bijection between isomorphism classes of framed $G$-bundles with framed connection over $M \times S^{1}$ and isomorphism classes of $\Omega G$ bundles with connection and Higgs field over $M$.

In both the free loop and based loop cases we call the $G$-bundle $\widetilde{P}$ the caloron transform of $P$.

In [13] we used the caloron correspondence to define characteristic classes for $\Omega G$-bundles in all odd degrees by using the Chern-Weil map for the corresponding $G$-bundle and integrating over the circle. Specifically, we have the following definition.

Definition 2.4 [13]. Suppose that $P \rightarrow M$ is an $\Omega G$-bundle with connection $A$ and Higgs field $\Phi$ and $\widetilde{P} \rightarrow M \times S^{1}$ is the caloron transform of $P$ with connection $\widetilde{A}$. Let $I^{k}(\mathfrak{g})$ be the set of all multilinear, symmetric, ad-invariant maps $\mathfrak{g} \times \cdots \times \mathfrak{g} \rightarrow \mathbb{R}$ and $c w: I^{k}(\mathfrak{g}) \rightarrow \Omega^{2 k}\left(M \times S^{1}\right)$ be the Chern-Weil map for the $G$-bundle $\widetilde{P}$, that is, $c w_{p}(\widetilde{A})=p(\widetilde{F}, \ldots, \widetilde{F})$ for the curvature $\widetilde{F}$ of $\widetilde{A}$. Then the string form of $p \in I^{k}(\mathfrak{g})$ is

$$
s_{p}(A, \Phi)=\int_{S^{1}} c w_{p}(\widetilde{A}) .
$$

The string form in terms of data on the $\Omega G$-bundle $P$ is given by

$$
s_{p}(A, \Phi)=k \int_{S^{1}} p(\nabla \Phi, F, \ldots, F) d \theta,
$$

where $F$ is the curvature of $A$. This can be seen by calculating the curvature $\widetilde{F}$ of the connection $\widetilde{A}=\operatorname{ad}\left(g^{-1}\right) A(\theta)+\Theta+\operatorname{ad}\left(g^{-1}\right) \Phi d \theta$ in terms of $F$ and $\Phi$,

$$
\widetilde{F}=\operatorname{ad}\left(g^{-1}\right)(F+\nabla \Phi d \theta),
$$

and substituting this into the formula for the string class in Definition 2.4. From now on we shall write $p\left(\nabla \Phi, F^{k-1}\right)$ for $p(\nabla \Phi, F, \ldots, F)$ and so on.

In [13] it is shown that $s_{p}(A, \Phi)$ is closed and independent of the choice of connection and Higgs field. We call the class of the form $s_{p}(A, \Phi)$ the string class of $P$ associated to $p$ and write $s_{p}(P) \in H^{2 k-1}(M)$. The main result of [13] is then the following theorem. 
THEOREM 2.5 [13]. If $P \rightarrow M$ is an $\Omega G$-bundle with caloron transform $\widetilde{P} \rightarrow$ $M \times S^{1}$ and

$$
s(P): I^{k}(\mathfrak{g}) \rightarrow H^{2 k-1}(M)
$$

is the map that associates to any invariant polynomial $p$ the string class of $P$, then the following diagram commutes.

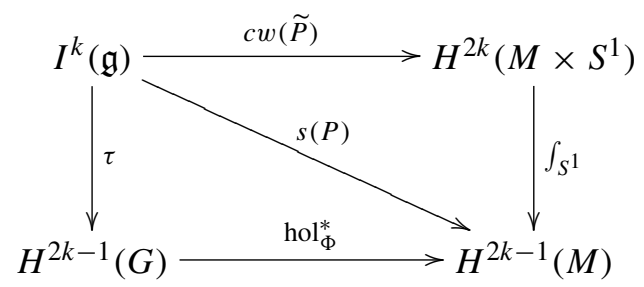

Here $\tau$ is the transgression map given by (see Section 6.1)

$$
\tau(p)=\left(-\frac{1}{2}\right)^{k-1} \frac{k !(k-1) !}{(2 k-1) !} p(\Theta,[\Theta, \Theta], \ldots,[\Theta, \Theta]),
$$

$\operatorname{cw}(\widetilde{P})$ is the usual Chern-Weil homomorphism for the G-bundle $\widetilde{P}$ and hol $_{\Phi}$ is the classifying map of $P$.

Note also that for the universal bundle, the map $s(P)$ is equal to the transgression map $\tau$.

\section{Classifying theory of $L G$-bundles}

3.1. The universal bundle. In order to extend the ideas from the previous section we need a model for $E L G$. This will allow us to calculate the universal string class. To construct this we view $L G$ as the semi-direct product $\Omega G \rtimes G$. The group multiplication is given by

$$
\left(\gamma_{1}, g_{1}\right)\left(\gamma_{2}, g_{2}\right)=\left(g_{2}^{-1} \gamma_{1} g_{2} \gamma_{2}, g_{1} g_{2}\right)
$$

and the isomorphism between $\Omega G \rtimes G$ and $L G$ is

$$
\Omega G \rtimes G \stackrel{\sim}{\rightarrow} L G ; \quad(\gamma, g) \mapsto g \gamma .
$$

On the level of Lie algebras, the isomorphism is

$$
\Omega \mathfrak{g} \rtimes \mathfrak{g} \stackrel{\sim}{\rightarrow} L \mathfrak{g} ; \quad(\xi, X) \mapsto X+\xi .
$$

We therefore need a model for the universal $\Omega G \rtimes G$-bundle. To find this, we shall take the product of the universal $\Omega G$-bundle and the universal $G$-bundle. Recall that a model for the universal $\Omega G$-bundle is given by the space of paths $p: \mathbb{R} \rightarrow G$ such that $p(0)=1$ and $p^{-1} \partial p$ is periodic. So, for our model for $E L G$ we shall take the space $P G \times E G$ which is contractible since $P G$ and $E G$ are both contractible. This is acted 
on by $\Omega G \rtimes G$ :

$$
(p, x)(\gamma, g)=\left(g^{-1} p g \gamma, x g\right)
$$

where $x g$ is the right action of $G$ on $E G$. This action is free and so $P G \times E G$ is a model for $E L G$ and $B L G$ is equal to $(P G \times E G) /(\Omega G \rtimes G)$. In fact, if we consider the map

$$
(P G \times E G) /(\Omega G \rtimes G) \rightarrow(G \times E G) / G ; \quad[p, x] \mapsto[p(2 \pi), x],
$$

where $[h, x]=\left[g^{-1} h g, x g\right]$, we can see this is well-defined, since

$$
[p, x]=\left[g^{-1} p g \gamma, x g\right] \mapsto\left[g^{-1} p(2 \pi) g \gamma(2 \pi), x g\right]=[p(2 \pi), x] .
$$

Furthermore, this is surjective, as the projection $P G \rightarrow G$ is surjective, and injective, for if we consider two elements $[p, x],[q, y] \in(P G \times E G) /(\Omega G \rtimes G)$ such that $[p(2 \pi), x]=[q(2 \pi), y]$ we have $y=x g$ and $q(2 \pi)=g^{-1} p(2 \pi) g$. That is, the paths $q$ and $g^{-1} p g$ have the same endpoint. Therefore, the path $g^{-1} p^{-1} g q$ is actually a based loop. And since $q=g^{-1} p g\left(g^{-1} p^{-1} g q\right)$, we have

$$
[q, y]=\left[g^{-1} p g \gamma, x g\right]=[p, x],
$$

where $\gamma=g^{-1} p^{-1} g q \in \Omega G$. Thus we have a diffeomorphism between $B L G$ and $(G \times E G) / G$, or simply $G \times{ }_{G} E G$. Importantly for our purposes this allows us to identify the cohomology of $B L G$ with the equivariant cohomology of $G$ with its adjoint action. That is,

$$
H^{*}(B L G)=H_{G}^{*}(G) .
$$

We will review equivariant cohomology in Section 4, as we will use it in the following to calculate the string classes for an $L G$-bundle.

3.2. Classifying maps. Given an $L G$-bundle $P \rightarrow M$, we can write down the classifying map of this bundle as follows. Choose a Higgs field $\Phi$ for $P$. Then define the map $f: P \rightarrow P G \times E G$ by

$$
f(q)=\left(\operatorname{hol}_{\Phi}(q), f_{G}(q)\right),
$$

where hol ${ }_{\Phi}$ is the Higgs field holonomy and $f_{G}$ is the classifying map for the $G$ bundle $P \times_{h} G \rightarrow M$, where $h: L G \rightarrow G$ is the homomorphism $h(\gamma)=\gamma(0)$. That is, $f(q)=(p, x)$ where $p^{-1} \partial p=\Phi(q)$ and $x$ is $f_{G}$ applied to the image of $q$ in $P \times{ }_{L G} G$. It is easy to see that this is equivariant with respect to the $L G$ action and hence descends to a map $M \rightarrow B L G$. For if $(\gamma, g) \in \Omega G \rtimes G$ then

$$
f(q(g \gamma))=\left(\operatorname{hol}_{\Phi}(q(g \gamma)), f_{G}(q) g\right)
$$

and so $f$ is equivariant in the $E G$ slot, by virtue of the fact that $f_{G}$ is a classifying map, and also in the $P G$ slot since if $\operatorname{hol}_{\Phi}(q)=p$ then

$$
\begin{aligned}
\Phi(q(g \gamma)) & =\operatorname{ad}\left((g \gamma)^{-1}\right) \Phi(q)+(g \gamma)^{-1} \partial(g \gamma) \\
& =\operatorname{ad}\left((g \gamma)^{-1}\right) \Phi(q)+\gamma^{-1} \partial \gamma
\end{aligned}
$$


and

$$
\begin{aligned}
(p(\gamma, g))^{-1} \partial(p(\gamma, g)) & =\left(g^{-1} p g \gamma\right)^{-1} \partial\left(g^{-1} p g \gamma\right) \\
& =\gamma^{-1} g^{-1} p^{-1} g\left(g^{-1} \partial p g \gamma+g^{-1} p g \partial \gamma\right) \\
& =\operatorname{ad}\left((g \gamma)^{-1}\right) p^{-1} \partial p+\gamma^{-1} \partial \gamma
\end{aligned}
$$

and so $\operatorname{hol}_{\Phi}(q(g \gamma))=p(\gamma, g)=\operatorname{hol}_{\Phi}(q)(g \gamma)$.

Note 6. Much of the construction above can be readily generalized to any group that is a semi-direct product. This is detailed in [19, Appendix B].

\section{Equivariant cohomology}

The results from the previous section imply that any generalization of Theorem 2.5 to the free loop group will necessarily involve equivariant cohomology. In particular, in the next section we shall calculate the universal string class for $L G$-bundles, in analogy with the corresponding calculation for the universal $\Omega G$-bundle in Section 2.2. This will be most conveniently represented as an equivariant differential form-an element of the so-called Cartan model of equivariant cohomology. In this section, therefore, we wish to review this theory. We shall mainly follow [7] (see also [2]).

4.1. The Borel model and the Weil model. Let $X$ be a manifold with an action of the Lie group $G$. If this action is free, for example, if $X$ is the total space of a principal $G$-bundle, then $X / G$ is a manifold and the equivariant cohomology of $X$ is given by the cohomology of the quotient: $H_{G}^{*}(X)=H^{*}(X / G)$. If the action is not free however, then the quotient may not be a manifold and the cohomology of $X / G$ may not be the correct object to study. In general the equivariant cohomology of $X$ is defined by $H_{G}^{*}(X):=H^{*}\left(X \times_{G} E\right)$, where $E$ is some contractible space on which $G$ acts freely. This definition is independent of the choice of the space $E$. A convenient example of such a space is of course the total space of the universal bundle, EG. So,

$$
H_{G}^{*}(X)=H^{*}\left(X \times_{G} E G\right) .
$$

This is called the Borel model for equivariant cohomology. The difficulty here is that in general it is not easy to study forms on $X \times_{G} E G$. In order to circumvent this we will introduce an algebraic version of the Borel model- the Weil model. Let us first set some conventions for the action of the Lie algebra $\mathfrak{g}$ on $\Omega(X)$.

Since $G$ acts on $X$, it acts on $\Omega(X)$ too. By convention, if $\psi: G \rightarrow \operatorname{Diff}(X)$ is a homomorphism, we define the action of $G$ on $\Omega(X)$ to be pull-back by the inverse of $\psi$. That is, $g \cdot \omega=\left(\psi_{g}^{-1}\right)^{*} \omega$. This is because $\psi$ satisfies $\psi_{g h}=\psi_{g} \psi_{h}$ whereas we would like to have a right action of $G$ on $X$ and $\Omega(X)$. Note that this means that the fundamental vector field generated by the Lie algebra element $\chi$ is given by

$$
\bar{\chi}_{x}:=\left.\frac{d}{d t}\right|_{0} \exp (-t \chi) \cdot x,
$$


for all $x \in X$. We shall write $L_{\chi}$ and $\iota_{\chi}$ for the Lie derivative and contraction with this vector field, respectively. Note that if $G$ acts freely on $X$ then a form $\omega \in \Omega(X)$ descends to the quotient $X / G$ precisely if it is invariant and horizontal with respect to the $G$ action. That is, if $L_{\chi} \omega$ and $\iota_{\chi} \omega$ both vanish for all $\chi \in \mathfrak{g}$. We call such a form basic. Alternatively, if $\left\{\xi_{i}\right\}$ is a basis for $\mathfrak{g}$ then $\omega$ descends if $L_{\xi_{i}} \omega=\iota_{\xi_{i}} \omega=0, i=1, \ldots, n$. From now on we shall write $L_{i}$ and $\iota_{i}$ for $L_{\xi_{i}}$ and $\iota_{\xi_{i}}$ respectively.

In order to rephrase the Borel model of equivariant cohomology in algebraic terms we shall use Weil's version of Chern-Weil theory, which first appeared in [4].

Define the Weil algebra to be the tensor product of the exterior algebra of $\mathfrak{g}^{*}$, the dual of $\mathfrak{g}$, with the symmetric algebra of $\mathfrak{g}^{*}$, that is, the graded algebra

$$
W:=\wedge\left(\mathfrak{g}^{*}\right) \otimes S\left(\mathfrak{g}^{*}\right) .
$$

Here the symmetric algebra is understood to be evenly graded, so every pure element is assigned twice its usual degree. It will be convenient to write down generators for $W$. Let $\left\{\theta^{i}\right\}$ be a basis for $\mathfrak{g}^{*}$. Then $W$ is generated by the elements $\theta^{i}$ of degree one in the exterior algebra and the corresponding elements, which we shall call $\mu^{i}$, of degree two in the symmetric algebra. The Weil algebra is in fact a differential graded algebra with the differential $d$ given on generators by

$$
\begin{aligned}
d \theta^{i} & =\mu^{i}-\frac{1}{2} c_{j k}^{i} \theta^{j} \theta^{k}, \\
d \mu^{i} & =c_{j k}^{i} \mu^{j} \theta^{k},
\end{aligned}
$$

where $c_{j k}^{i}$ are the structure constants of $\mathfrak{g}$. We consider the cohomology of $W$ with respect to this differential.

It is easy to see [7] that $S\left(\mathfrak{g}^{*}\right)$ is the horizontal part of $W$ and so the basic subalgebra of $W$-the invariant part of this-is just the invariant, symmetric polynomials in $\mathfrak{g}^{*}$. This gives rise to the Chern-Weil homomorphism (see, for example, [7] or [4] for the original exposition).

Note that we can obtain the generators $\theta^{i}$ and $\mu^{i}$ for $W$ via a connection $a$ on $E G$ and its curvature $f$. For connections on universal bundles, see for example $[15,16,18]$ or [5] for the simplicial point of view.

Recall that the de Rham complex $\Omega(X)$ forms a graded algebra itself. Here is the standard result.

THEOREM 4.1 (Equivariant de Rham theorem). The equivariant cohomology of $X$ is given by the basic cohomology, that is, the cohomology of the basic subcomplex, of $W \otimes \Omega(X):$

$$
H_{G}^{*}(X)=H_{\text {basic }}^{*}(W \otimes \Omega(X)) .
$$

The basic cohomology of the differential graded algebra $W \otimes \Omega(X)$ is called the Weil model of the equivariant cohomology of $X$.

The connection-curvature construction above helps translate between the Borel model and the Weil model. However, it is still quite difficult to perform computations 
with the Weil model. Therefore, we shall present another model in the next sectioncalled the Cartan model—and describe the method for translating between this model and the Weil model: the Mathai-Quillen isomorphism.

4.2. The Cartan model and the Mathai-Quillen isomorphism. In order to perform calculations with equivariant cohomology it is useful to find an automorphism of the Weil model, which simplifies things significantly. Define the degree-zero endomorphism $\gamma \in \operatorname{End}(W \otimes \Omega(X))$ by

$$
\gamma=\theta^{i} \otimes \iota_{i}
$$

Clearly this is nilpotent and so the automorphism

$$
\phi:=\exp \gamma
$$

is a finite sum. This automorphism is known as the Mathai-Quillen isomorphism. The following fundamental observation, due to Mathai and Quillen [11] and Kalkman [9], is proved (in the form below) in [7].

THEOREM 4.2. The Mathai-Quillen isomorphism $\phi$ carries the horizontal subspace $(W \otimes \Omega(X))_{\text {hor }}$ into $W_{\text {hor }} \otimes \Omega(X)=S\left(\mathfrak{g}^{*}\right) \otimes \Omega(X)$ and hence the basic subcomplex $(W \otimes \Omega(X))_{\text {basic }}$ into the invariant elements of $S\left(\mathfrak{g}^{*}\right) \otimes \Omega(X)$, denoted $\left(S\left(\mathfrak{g}^{*}\right) \otimes\right.$ $\Omega(X))^{G}$. On this basic subcomplex the differential is transformed into $1 \otimes d-\mu^{i} \otimes \iota_{i}$.

Definition 4.3. The cohomology of the complex $\Omega_{G}(X):=\left(S\left(\mathfrak{g}^{*}\right) \otimes \Omega(X)\right)^{G}$ with the differential above, $1 \otimes d-\mu^{i} \otimes \iota_{i}$, is called the Cartan model for the equivariant cohomology of $X$. The elements of the space $\Omega_{G}(X)$ are called equivariant differential forms.

Theorem 4.2 implies that the equivariant cohomology according to the Cartan model agrees with that of the Weil model. The elements of $\Omega_{G}(X)$ have a pleasant interpretation as form-valued polynomials on $\mathfrak{g}^{*}$. The invariance condition translates with this interpretation into equivariance of the polynomials. The differential on an element $\omega$ is then given by

$$
d(\omega(\chi))-\iota_{\chi}(\omega(\chi))
$$

REMARK 4.4. Suppose that $\omega$ is a degree $n$ basic element of the Weil algebra. Then $\omega$ is a sum of terms, each lying in a different part of the graded algebra

$$
\bigoplus_{i+2 j+k=n} \wedge^{i}\left(\mathfrak{g}^{*}\right) \otimes S^{j}\left(\mathfrak{g}^{*}\right) \otimes \Omega^{k}(X)
$$

Further,

$$
\begin{gathered}
\gamma: \wedge^{i}\left(\mathfrak{g}^{*}\right) \otimes S^{j}\left(\mathfrak{g}^{*}\right) \otimes \Omega^{k}(X) \rightarrow \wedge^{i+1}\left(\mathfrak{g}^{*}\right) \otimes S^{j}\left(\mathfrak{g}^{*}\right) \otimes \Omega^{k-1}(X), \\
\gamma^{2}: \wedge^{i}\left(\mathfrak{g}^{*}\right) \otimes S^{j}\left(\mathfrak{g}^{*}\right) \otimes \Omega^{k}(X) \rightarrow \wedge^{i+2}\left(\mathfrak{g}^{*}\right) \otimes S^{j}\left(\mathfrak{g}^{*}\right) \otimes \Omega^{k-2}(X)
\end{gathered}
$$


and so on. However, since $\omega$ is basic, we know that $\phi(\omega) \in\left(S\left(\mathfrak{g}^{*}\right) \otimes \Omega(X)\right)^{G}$, that is, it has no components in $\wedge\left(\mathfrak{g}^{*}\right)$. Therefore, since $\phi=1+\gamma+\frac{1}{2} \gamma^{2}+\cdots$ and every application of $\gamma$ raises the degree of the exterior algebra by one, the effect of $\phi$ is essentially to 'pick out' the components of $\omega$ which have no component in $\wedge\left(\mathfrak{g}^{*}\right)$. The end result of $\phi$ acting on the rest of the terms is that they all must cancel.

\section{The universal string class}

Now that we have a model for the universal $L G$-bundle we would like to prove the analogue of Theorem 2.5 for $L G$-bundles. Note that this will naturally involve equivariant cohomology since rather than the universal string class being equal to the transgression map, which takes values in $H^{*}(G)=H^{*}(B \Omega G)$, the universal string class for $L G$-bundles will be in $H^{*}\left(G \times{ }_{G} E G\right)=H_{G}^{*}(G)$. Thus the first thing we need to do is calculate this universal string class. To illustrate the idea we will do this in the degree-three case first and extend the result to higher degrees in the next section.

5.1. The string class in the Borel model. Firstly we will use Theorem 2.1 to calculate a differential form representing the string class of $E L G=P G \times E G$. Note that this will give us a class in $H^{*}\left(G \times{ }_{G} E G\right)$, the Borel model for the equivariant cohomology of $G$, where $G$ acts on itself by conjugation. In order to use Theorem 2.1, the first thing we need is a connection on $P G \times E G$. Now $P G$ is already a smooth manifold. In order to define a smooth structure and find a connection on $E G$ we use the results in $[15,16,18]$. As long as the dimension of the base of the $G$-bundle $P \rightarrow M$ is less than or equal to $n$ this gives a construction of a smooth bundle $E G_{n} \rightarrow B G_{n}$ with connection, which is a model for the universal $G$-bundle. From now on we assume therefore that the dimension of the base of our $L G$-bundle is fixed, and less than or equal to $n$ for some $n$.

To define a connection we need to know what a vertical vector looks like. Consider the vector in $T_{(p, x)}\left(P G \times E G_{n}\right)=T_{p} P G \times T_{x} E G_{n}$ generated by the Lie algebra element $(\xi, X) \in \Omega \mathfrak{g} \rtimes \mathfrak{g}$ :

$$
\begin{aligned}
\overline{(\xi, X)}_{(p, x)} & :=\left.\frac{d}{d t}\right|_{0}\left((1-t X) p(1+t X)(1+t \xi), x e^{t X}\right) \\
& =\left.\frac{d}{d t}\right|_{0}\left(t(-X p+p X+p \xi), x e^{t X}\right) \\
& =\left(p\left(X-\operatorname{ad}\left(p^{-1}\right) X+\xi\right), \bar{X}_{x}\right) .
\end{aligned}
$$

(Here $\bar{X}_{x}$ is the vertical vector generated by the Lie algebra element $X$ at $x$.)

A connection is given [19] by

$$
A=\Theta-\alpha \operatorname{ad}\left(p^{-1}\right)\left\{\operatorname{ev}_{2 \pi}^{*} \widehat{\Theta}-\operatorname{ad}(p(2 \pi)) a+a\right\}+\operatorname{ad}\left(p^{-1}\right) a .
$$

As before, $\Theta$ is the Maurer-Cartan form, $\widehat{\Theta}$ is the right Maurer-Cartan form, $\alpha$ is a smooth function such that $\alpha(t)=0$ for all $t \leq 0$ and $\alpha(t)=1$ for all $t \geq 2 \pi$ 
and $\mathrm{ev}_{2 \pi}: P G \rightarrow G$ is the projection. The form $a$ is a connection on $E G$, which we shall assume that we have using the results cited earlier. It can be easily checked that this form returns the Lie algebra element $\xi+X \in L \mathfrak{g}$ when evaluated on the vertical vector above and also that it transforms in the adjoint representation. Thus it satisfies the conditions for a connection.

To calculate the string class we will need the curvature of this connection and a Higgs field. As usual, the curvature is given by the formula

$$
F=D A
$$

where $D$ is the covariant exterior derivative. Hence we have

$$
F\left((V, W),\left(V^{\prime}, W^{\prime}\right)\right)=-\frac{1}{2} A\left(\left[h(V, W), h\left(V^{\prime}, W^{\prime}\right)\right]\right)
$$

where $h X$ is the projection onto the horizontal subspace of the vector $X$. This yields

$$
\begin{aligned}
F=( & \left.\alpha^{2}-\alpha\right) \operatorname{ad}\left(p^{-1}\right)\left\{\frac{1}{2}\left[\operatorname{ev}_{2 \pi}^{*} \widehat{\Theta}, \operatorname{ev}_{2 \pi}^{*} \widehat{\Theta}\right]-\left[\operatorname{ev}_{2 \pi}^{*} \widehat{\Theta}, \operatorname{ad}\left(p(2 \pi)^{-1}\right) a\right]\right. \\
& \left.+\left[\operatorname{ev}_{2 \pi}^{*} \widehat{\Theta}, a\right]+\frac{1}{2} \operatorname{ad}(p(2 \pi))[a, a]-[\operatorname{ad}(p(2 \pi)) a, a]+\frac{1}{2}[a, a]\right\} \\
& +\alpha \operatorname{ad}\left(p^{-1}\right)(\operatorname{ad}(p(2 \pi)) f-f)+\operatorname{ad}\left(p^{-1}\right) f
\end{aligned}
$$

where $f$ is the curvature of $a$.

The other piece of data we need to calculate the string class is a Higgs field for $E L G$. Define the map

$$
\Phi: P G \times E G_{n} \rightarrow L \mathfrak{g}
$$

by

$$
\Phi(p, x)=p^{-1} \partial p
$$

Then by the calculation at the end of Section 3.2 we see that $\Phi$ is a Higgs field for $P G \times E G_{n}$. Next we need to calculate

$$
\nabla \Phi=d \Phi+[A, \Phi]-\partial A
$$

We can show

$$
d \Phi=[\Phi, \Theta]+\partial \Theta
$$

and so

$$
\begin{aligned}
\nabla \Phi=[\Phi, \Theta]+\partial \Theta \\
+\left[\Theta-\alpha \operatorname{ad}\left(p^{-1}\right)\left\{\operatorname{ev}_{2 \pi}^{*} \widehat{\Theta}-\operatorname{ad}(p(2 \pi)) a+a\right\}+\operatorname{ad}\left(p^{-1}\right) a, \Phi\right] \\
\quad-\partial\left(\Theta-\alpha \operatorname{ad}\left(p^{-1}\right)\left\{\operatorname{ev}_{2 \pi}^{*} \widehat{\Theta}-\operatorname{ad}(p(2 \pi)) a+a\right\}+\operatorname{ad}\left(p^{-1}\right) a\right) \\
=\partial \alpha \operatorname{ad}\left(p^{-1}\right)\left\{\operatorname{ev}_{2 \pi}^{*} \widehat{\Theta}-\operatorname{ad}(p(2 \pi)) a+a\right\} .
\end{aligned}
$$


Therefore, using the formula from Theorem 2.1, the string class for $P G \times E G_{n}$ as a class in $H^{*}\left(G \times{ }_{G} E G\right)$ is

$$
\begin{aligned}
-\frac{1}{4 \pi^{2}} \int_{S^{1}}\left\langle( \alpha ^ { 2 } - \alpha ) \left(\frac{1}{2}\left[\mathrm{ev}_{2 \pi}^{*} \widehat{\Theta}, \mathrm{ev}_{2 \pi}^{*} \widehat{\Theta}\right]-\left[\mathrm{ev}_{2 \pi}^{*} \widehat{\Theta}, \operatorname{ad}\left(p(2 \pi)^{-1}\right) a\right]\right.\right. \\
\left.+\left[\mathrm{ev}_{2 \pi}^{*} \widehat{\Theta}, a\right]+\frac{1}{2} \operatorname{ad}(p(2 \pi))[a, a]-[\operatorname{ad}(p(2 \pi)) a, a]+\frac{1}{2}[a, a]\right) \\
\left.+\alpha \operatorname{ad}\left(p^{-1}\right)(\operatorname{ad}(p(2 \pi)) f-f)+\operatorname{ad}\left(p^{-1}\right) f, \partial \alpha\left(\mathrm{ev}_{2 \pi}^{*} \widehat{\Theta}-\operatorname{ad}(p(2 \pi)) a+a\right)\right\rangle \\
=-\frac{1}{8 \pi^{2}}\left\langle-\frac{1}{6}[\widehat{\Theta}, \widehat{\Theta}]+\frac{1}{3}[\widehat{\Theta}, \operatorname{ad}(p(2 \pi)) a]\right. \\
\quad-\frac{1}{3}[\widehat{\Theta}, a]+\frac{1}{6} \operatorname{ad}(p(2 \pi))[a, a]+\frac{1}{3}[\operatorname{ad}(p(2 \pi)) a, a] \\
\left.\quad-\frac{1}{6}[a, a]+\operatorname{ad} p(2 \pi) f+f, \widehat{\Theta}-\operatorname{ad} p(2 \pi) a+a\right\rangle \\
=\frac{1}{8 \pi^{2}}\left(\frac{1}{6}\langle[\widehat{\Theta}, \widehat{\Theta}], \widehat{\Theta}\rangle-\frac{1}{2}\langle[\widehat{\Theta}, \widehat{\Theta}], \operatorname{ad}(g) a\rangle+\frac{1}{2}\langle[\widehat{\Theta}, \widehat{\Theta}], a\rangle\right. \\
+\frac{1}{2}\langle\widehat{\Theta}, \operatorname{ad}(g)[a, a]\rangle-\langle\widehat{\Theta},[\operatorname{ad}(g) a, a]\rangle+\frac{1}{2}\langle\widehat{\Theta},[a, a]\rangle \\
+\frac{1}{2}\langle a, \operatorname{ad}(g)[a, a]\rangle-\frac{1}{2}\langle\operatorname{ad}(g) a,[a, a]\rangle-\langle\operatorname{ad}(g) f+f, \widehat{\Theta}\rangle \\
\left.\quad-\left\langle\operatorname{ad}(g) f-\operatorname{ad}\left(g^{-1}\right) f, a\right\rangle\right)
\end{aligned}
$$

where $g=p(2 \pi)$. Note that although there is some freedom in the choice of $\alpha$, we have $\int_{S^{1}}\left(\alpha^{2}-\alpha\right) \partial \alpha=-\frac{1}{6}$ and the result is independent of $\alpha$.

5.2. The string class in the Cartan model. The formula (5.1) above for the string class is quite unwieldy. We have already seen that the most compact representation for equivariant cohomology classes is via equivariant differential forms. We shall now proceed to find an equivariant differential form representing the same class as above. Firstly, let us write this form as an element of the Weil model. Recall that this will be a sum of terms lying in different parts of the graded algebra $W$. The degree-three component of the Weil model is given by

$$
\begin{aligned}
(W \otimes \Omega(G))^{3}= & \bigoplus_{i+2 j+k=3} \wedge^{i}\left(\mathfrak{g}^{*}\right) \otimes S^{j}\left(\mathfrak{g}^{*}\right) \otimes \Omega^{k}(G) \\
= & \left(\wedge^{0} \otimes S^{0} \otimes \Omega^{3}\right) \oplus\left(\wedge^{1} \otimes S^{0} \otimes \Omega^{2}\right) \oplus\left(\wedge^{2} \otimes S^{0} \otimes \Omega^{1}\right) \\
& \oplus\left(\wedge^{3} \otimes S^{0} \otimes \Omega^{0}\right) \oplus\left(\wedge^{0} \otimes S^{1} \otimes \Omega^{1}\right) \oplus\left(\wedge^{1} \otimes S^{1} \otimes \Omega^{0}\right) .
\end{aligned}
$$

We can use the connection-curvature construction from Section 4.1 to rewrite the string class as follows. Consider, for example, the terms $-\frac{1}{2}\langle[\widehat{\Theta}, \widehat{\Theta}], \operatorname{ad}(g) a\rangle$ 
and $\frac{1}{2}\langle[\widehat{\Theta}, \widehat{\Theta}], a\rangle$ above. In the Weil model, these terms should live in $\wedge^{1} \otimes S^{0} \otimes \Omega^{2}$. If we expand the connection in terms of a basis $\left\{\xi_{i}\right\}$ for $\mathfrak{g}$ then we can write

$$
\begin{aligned}
\frac{1}{2}\langle[\widehat{\Theta}, \widehat{\Theta}], a\rangle-\frac{1}{2}\langle[\widehat{\Theta}, \widehat{\Theta}], \operatorname{ad}(g) a\rangle & =\frac{1}{2}\left\langle[\widehat{\Theta}, \widehat{\Theta}], a^{i} \xi_{i}\right\rangle-\frac{1}{2}\left\langle[\widehat{\Theta}, \widehat{\Theta}], \operatorname{ad}(g) a^{i} \xi_{i}\right\rangle \\
& =\frac{1}{2} a^{i}\left\langle[\widehat{\Theta}, \widehat{\Theta}], \xi_{i}-\operatorname{ad}(g) \xi_{i}\right\rangle .
\end{aligned}
$$

Therefore the element in $\wedge^{1} \otimes S^{0} \otimes \Omega^{2}$ corresponding to these two terms is

$$
\frac{1}{2} a^{i}\left\langle[\widehat{\Theta}, \widehat{\Theta}], \xi_{i}-\operatorname{ad}(g) \xi_{i}\right\rangle \text {. }
$$

Similarly, for the terms with the connection appearing twice (the middle three terms), we have

$$
\frac{1}{2} a^{i} \wedge a^{j}\left\langle\hat{\Theta}, c_{i j}^{k}\left(\xi_{k}+\operatorname{ad}(g) \xi_{k}\right)\right\rangle-a^{i} \wedge a^{j}\left\langle\hat{\Theta},\left[\operatorname{ad}(g) \xi_{i}, \xi_{j}\right]\right\rangle \in \wedge^{2} \otimes S^{0} \otimes \Omega^{1} .
$$

The last two terms in (5.1) live in $\wedge^{0} \otimes S^{1} \otimes \Omega^{1}$ and $\wedge^{1} \otimes S^{1} \otimes \Omega^{0}$ respectively, and we view them as the $\Omega^{1}$-valued ( $\wedge^{1}$-valued) polynomials on $\mathfrak{g}$

$$
\chi \mapsto-\langle\operatorname{ad}(g) \chi+\chi, \hat{\Theta}\rangle \quad \text { and } \quad \chi \mapsto-a^{i}\left\langle\operatorname{ad}(g) \chi-\operatorname{ad}\left(g^{-1}\right) \chi, \xi_{i}\right\rangle .
$$

We can now apply the Mathai-Quillen isomorphism to the string class $s$, interpreted as above. That is, we can calculate $\phi(s)=(\exp \gamma)(s)=s+\gamma(s)+\cdots$. Note that in light of Remark 4.4 we can already identify the image of $s$ under $\phi$ since we know that it is a basic form. Indeed we are viewing it as a form on $G \times E G$ which descends to $G \times{ }_{G} E G$. However, we shall present the calculation here as it illustrates the beauty of Mathai and Quillen's result and Cartan's formalism. We will calculate a few terms here and leave the rest as an exercise. We write $\omega_{i j k}$ for the $\wedge^{i} \otimes S^{j} \otimes \Omega^{k}$ part of the element $\omega \in W \otimes \Omega(G)$. Let us begin with the term $\frac{1}{6}\langle[\hat{\Theta}, \hat{\Theta}], \hat{\Theta}\rangle=: s_{003}$. Recalling that the $a^{i}$ are the connection elements in $W$,

$$
\begin{aligned}
\gamma\left(s_{003}\right) & =\frac{1}{6}\left(a^{i} \otimes \iota_{i}\right)(\langle[\hat{\Theta}, \hat{\Theta}], \hat{\Theta}\rangle) \\
& =\frac{1}{2} a^{i}\left\langle\left[\iota_{i} \hat{\Theta}, \hat{\Theta}\right], \hat{\Theta}\right\rangle .
\end{aligned}
$$

Now recall that here $\iota_{i}$ means contraction with the vector field generating the action of $G$ on itself-in this case the adjoint action. So $\iota_{\chi} \widehat{\Theta}$ is given by

$$
\hat{\Theta}_{g}\left(\left.\frac{d}{d t}\right|_{0} \exp (-t \chi) g \exp (t \chi)\right)=\hat{\Theta}_{g}(g \chi-\chi g)=\operatorname{ad}(g) \chi-\chi .
$$

Therefore,

$$
\gamma\left(s_{003}\right)=\frac{1}{2} a^{i}\left\langle[\hat{\Theta}, \hat{\Theta}], \operatorname{ad}(g) \xi_{i}-\xi_{i}\right\rangle .
$$

Notice that the term $s_{102}:=\left.s\right|_{\wedge^{1} \otimes S^{0} \otimes \Omega^{2}}=\frac{1}{2} a^{i}\left\langle[\hat{\Theta}, \hat{\Theta}], \xi_{i}-\operatorname{ad}(g) \xi_{i}\right\rangle$ cancels out. We can calculate the $\wedge^{2} \otimes S^{0} \otimes \Omega^{1}$ part of $\phi(s)$ using

$$
\begin{aligned}
s_{201} & =\frac{1}{2} a^{i} \wedge a^{j}\left\langle\hat{\Theta}, c_{i j}^{k}\left(\xi_{k}+\operatorname{ad}(g) \xi_{k}\right)\right\rangle-a^{i} \wedge a^{j}\left\langle\hat{\Theta},\left[\operatorname{ad}(g) \xi_{i}, \xi_{j}\right]\right\rangle, \\
\gamma\left(s_{102}\right) & =-a^{i} \wedge a^{j}\left\langle\hat{\Theta}, c_{i j}^{k}\left(\xi_{k}+\operatorname{ad}(g) \xi_{k}\right)\right\rangle+2 a^{i} \wedge a^{j}\left\langle\hat{\Theta},\left[\operatorname{ad}(g) \xi_{i}, \xi_{j}\right]\right\rangle=-2 s_{201}, \\
\frac{1}{2} \gamma^{2}\left(s_{003}\right) & =\frac{1}{2} a^{i} \wedge a^{j}\left\langle\hat{\Theta}, c_{i j}^{k}\left(\xi_{k}+\operatorname{ad}(g) \xi_{k}\right)\right\rangle-a^{i} \wedge a^{j}\left\langle\hat{\Theta},\left[\operatorname{ad}(g) \xi_{i}, \xi_{j}\right]\right\rangle=s_{201} .
\end{aligned}
$$


We can calculate all the other terms similarly and we find that everything cancels, as indeed it must, except for the terms in $\wedge^{0} \otimes S^{0} \otimes \Omega^{3}$ and $\wedge^{0} \otimes S^{1} \otimes \Omega^{1}$. These are given by $\frac{1}{6}\langle[\hat{\Theta}, \hat{\Theta}], \hat{\Theta}\rangle$ and $\chi \mapsto-\langle\chi, \Theta+\hat{\Theta}\rangle$. Therefore, we find the following theorem holds.

THEOREM 5.1. The universal string class for LG-bundles is represented in $H_{G}^{3}(G)$ by the class of the equivariant differential form

$$
\frac{1}{8 \pi^{2}}\left(\frac{1}{6}\langle[\hat{\Theta}, \hat{\Theta}], \hat{\Theta}\rangle-\langle\chi, \Theta+\hat{\Theta}\rangle\right) .
$$

REMARK 5.2. The equivariant form above coincides with the equivariant extension of the transgression form $\left(48 \pi^{2}\right)^{-1}\langle\hat{\Theta},[\hat{\Theta}, \hat{\Theta}]\rangle$ defined by Alekseev and Meinrenken [1]. There are also equivariant extensions of the higher analogues of these forms and in the next section we shall show that these represent the universal string classes in all odd dimensions.

\section{Higher string classes for $L G$-bundles}

We would now like to extend Theorem 5.1 to string classes in all odd degrees, in analogy with the results for $\Omega G$-bundles from [13]. As remarked at the end of the last section we wish to show that these coincide with the classes of the equivariant transgression forms as defined in $[1,8]$. Therefore, we first review the construction of these forms.

6.1. Equivariant transgression forms. Let $p \in I^{k}(\mathfrak{g})$ and consider the 1-form $t \Theta$ on $G \times[0,1]$. Define the 'curvature' $F_{t}$ of this form by $d(t \Theta)+\frac{1}{2}[t \Theta, t \Theta]$, and consider the $(2 k-1)$-form on $G$ given by

$$
\begin{aligned}
-\int_{0}^{1} p\left(F_{t}^{k}\right) & =k \int_{0}^{1} p\left(\Theta,\left(\frac{1}{2}\left(t^{2}-t\right)[\Theta, \Theta]\right)^{k-1}\right) d t \\
& =\left(-\frac{1}{2}\right)^{k-1} \frac{k !(k-1) !}{(2 k-1) !} p\left(\Theta,[\Theta, \Theta]^{k-1}\right)
\end{aligned}
$$

It is easy to see that this form is closed: we call it the transgression of $p$. We write $\tau(p)$ for both the $(2 k-1)$-form and its class in $H^{2 k-1}(G)$.

To define the equivariant version of the form above, consider the 'equivariant curvature' of the 1 -form above, $F_{G}(t \Theta)(\chi)$, given by $d_{G}(t \Theta)(\chi)+\frac{1}{2}[t \Theta, t \Theta]$ where $d_{G}=d-\iota_{\chi}$ is the equivariant differential from the Cartan model (Definition 4.3). The equivariant transgression of $p$ is given by $[1,8]$

$$
\tau_{G}(p)=-\int_{0}^{1} p\left(\left(F_{G}(t \Theta)(\chi)+\chi\right)^{k}\right) .
$$

We have

$$
F_{G}(t \Theta)=d t \wedge \Theta+\frac{1}{2}\left(t^{2}-t\right)[\Theta, \Theta]-t\left(\chi-\operatorname{ad}\left(g^{-1}\right) \chi\right)
$$


and so

$$
\begin{aligned}
\tau_{G}(p) & =-\int_{0}^{1} p\left(\left(d t \wedge \Theta+\frac{1}{2}\left(t^{2}-t\right)[\Theta, \Theta]-t\left(\chi-\operatorname{ad}\left(g^{-1}\right) \chi\right)+\chi\right)^{k}\right) \\
& =k \int_{0}^{1} p\left(\Theta,\left(\frac{1}{2}\left(t^{2}-t\right)[\Theta, \Theta]+(1-t) \chi+t \operatorname{ad}\left(g^{-1}\right) \chi\right)^{k-1}\right) d t
\end{aligned}
$$

It is easy to see that $\tau_{G}(p)$ is equivariantly closed and so defines a class in $H_{G}^{2 k-1}(G)$.

6.2. Higher string classes for $\boldsymbol{L} \boldsymbol{G}$-bundles. Let $P \rightarrow M$ be an $L G$-bundle with connection $A$ and Higgs field $\Phi$ and denote by $F$ the curvature of $A$ and by $\nabla \Phi=$ $d \Phi+[A, \Phi]-\partial A$ the covariant derivative of $\Phi$. Following [13] we define the string form of the pair $(A, \Phi)$ associated to $p \in I^{k}(\mathfrak{g})$ by

$$
s_{p}(A, \Phi)=\int_{S^{1}} c w_{p}(\widetilde{A})
$$

where $\widetilde{A}$ is the connection on the caloron transform of $P$. As in Section 2.3, we have the following formula for the string form:

$$
s_{p}(A, \Phi)=k \int_{S^{1}} p\left(\nabla \Phi, F^{k-1}\right) d \theta .
$$

We also have the results analogous to [13].

PROPOSITION 6.1. The string form is closed and so defines a cohomology class in $H^{2 k-1}(M)$.

This class, which we denote by $s_{p}(P)$, is called the string class of $P$ associated to $p$.

PROPOSITION 6.2. The string class is independent of the choice of connection and Higgs field.

Proposition 6.3. The string class is natural, that is, $s_{p}\left(f^{*} P\right)=f^{*} s_{p}(P)$ if $f$ : $N \rightarrow M$. In particular, $s_{p}$ defines a characteristic class for $L G$-bundles.

The proofs of Propositions 6.1, 6.2 and 6.3 are all identical to the $\Omega G$ case.

As in Section 5, the string class associated to $p \in I^{k}(G)$ of the universal $L G$ bundle is an equivariant cohomology class. We have the following result concerning this class.

PROPOSITION 6.4. The string class associated to $p \in I^{k}(\mathfrak{g})$ of the universal LGbundle is represented in $H_{G}^{2 k-1}(G)$ by the equivariant transgression of $p$. That is,

$$
s_{p}(E L G)=\tau_{G}(p) .
$$


PROOF. Recall that a connection and Higgs field for the universal bundle were given in Section 5.1. The curvature of this connection was

$$
\begin{aligned}
F=( & \left.\alpha^{2}-\alpha\right) \operatorname{ad}\left(p^{-1}\right)\left(\frac{1}{2}\left[\operatorname{ev}_{2 \pi}^{*} \widehat{\Theta}, \operatorname{ev}_{2 \pi}^{*} \widehat{\Theta}\right]-\left[\operatorname{ev}_{2 \pi}^{*} \widehat{\Theta}, \operatorname{ad}\left(p(2 \pi)^{-1}\right) a\right]\right. \\
& \left.+\left[\operatorname{ev}_{2 \pi}^{*} \widehat{\Theta}, a\right]+\frac{1}{2} \operatorname{ad}(p(2 \pi))[a, a]-[\operatorname{ad}(p(2 \pi)) a, a]+\frac{1}{2}[a, a]\right) \\
& +\alpha \operatorname{ad}\left(p^{-1}\right)(\operatorname{ad}(p(2 \pi)) f-f)+\operatorname{ad}\left(p^{-1}\right) f,
\end{aligned}
$$

where $a$ is a connection on $E G$ and $f$ is its curvature. The covariant derivative of the Higgs field was given by

$$
\nabla \Phi=\partial \alpha \operatorname{ad}\left(p^{-1}\right)\left(\mathrm{ev}_{2 \pi}^{*} \widehat{\Theta}-\operatorname{ad}(p(2 \pi)) a+a\right) .
$$

We wish to calculate the equivariant differential form representing the string class

$$
s_{p}=k \int_{S^{1}} p\left(\nabla \Phi, F^{k-1}\right) d \theta ;
$$

we omit the dependence on the $L G$-bundle since this is the universal class. Recall that in order to do this we use the Mathai-Quillen isomorphism, which, according to Remark 4.4, requires us to ignore any terms in $s_{p}$ which involve the connection $a$. Therefore we see that the Mathai-Quillen isomorphism applied to the string class gives the equivariant form

$$
\begin{aligned}
s_{p} & =k \int_{S^{1}} p\left(\widehat{\Theta},\left(\frac{1}{2}\left(\alpha^{2}-\alpha\right)[\hat{\Theta}, \hat{\Theta}]+\alpha(\operatorname{ad}(g) \chi-\chi)+\chi\right)^{k-1}\right) \partial \alpha d \theta \\
& =k \int_{0}^{1} p\left(\Theta,\left(\frac{1}{2}\left(\alpha^{2}-\alpha\right)[\Theta, \Theta]+\alpha\left(\chi-\operatorname{ad}\left(g^{-1}\right) \chi\right)+\operatorname{ad}\left(g^{-1}\right) \chi\right)^{k-1}\right) d \alpha \\
& =k \int_{0}^{1} p\left(\Theta,\left(\frac{1}{2}\left(\alpha^{2}-\alpha\right)[\Theta, \Theta]+\alpha \chi+(1-\alpha) \operatorname{ad}\left(g^{-1}\right) \chi\right)^{k-1}\right) d \alpha .
\end{aligned}
$$

Now making the change of variables $\alpha \mapsto 1-t$ gives the required result.

We now have the result we were looking for, in analogy with Theorem 2.5.

TheOREM 6.5. Let $P \rightarrow M$ be an LG-bundle and $\widetilde{P} \rightarrow M \times S^{1}$ its caloron transform. If

$$
s(P): I^{k}(\mathfrak{g}) \rightarrow H^{2 k-1}(M)
$$

is the map that gives for any $p \in I^{k}(\mathfrak{g})$ its associated string class, then the following diagram commutes

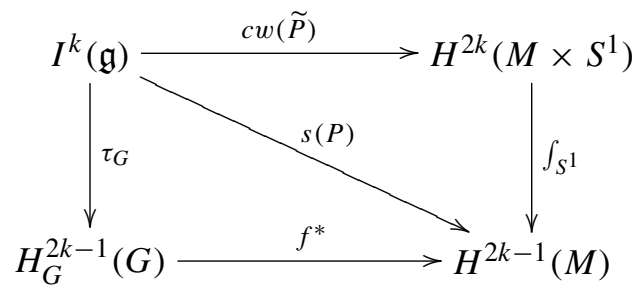

Here $f$ is the classifying map of $P$ (Section 3.2). 
PROOF. The upper triangle in the diagram commutes by the definition of $s(P)$. Proposition 6.3 tells us that the string class is natural, so for the lower triangle it is enough to calculate the universal string class and then $s_{p}(P)=f^{*} s_{p}(E L G)$. But we know from Proposition 6.4 that $s_{p}(E L G)$ is equal to the equivariant transgression $\tau_{G}(p)$. Therefore, the diagram commutes.

\section{Acknowledgement}

The author thanks Michael Murray and Mathai Varghese for useful discussions, and the referees for many useful suggestions.

\section{References}

[1] A. Alekseev and E. Meinrenken, 'The Atiyah algebroid of the path fibration over a Lie group', Lett. Math. Phys. 90 (2009), 23-58.

[2] M. F. Atiyah and R. Bott, 'The moment map and equivariant cohomology', Topology 23(1) (1984), $1-28$.

[3] A. L. Carey and J. Mickelsson, 'The universal gerbe, Dixmier-Douady class, and gauge theory', Lett. Math. Phys. 59(1) (2002), 47-60.

[4] H. Cartan, 'Notions d'algèbre différentielle; application aux groupes de Lie et aux variétés où opère un groupe de Lie', in: Colloque de topologie (espaces fibrés), Bruxelles, 1950 (Georges Thone, Liège, 1951), pp. 15-27.

[5] J. L. Dupont, Curvature and Characteristic Classes, Lecture Notes in Mathematics, 640 (Springer, Berlin, 1978).

[6] H. Garland and M. K. Murray, 'Kac-Moody monopoles and periodic instantons', Comm. Math. Phys. 120(2) (1988), 335-351.

[7] V. W. Guillemin and S. Sternberg, Supersymmetry and Equivariant de Rham Theory, Mathematics Past and Present (Springer, Berlin, 1999), With an appendix containing two reprints by Henri Cartan.

[8] L. C. Jeffrey, 'Group cohomology construction of the cohomology of moduli spaces of flat connections on 2-manifolds', Duke Math. J. 77(2) (1995), 407-429.

[9] J. Kalkman, 'A BRST model applied to symplectic geometry', PhD Thesis, Universiteit Utrecht, 1993.

[10] T. P. Killingback, 'World-sheet anomalies and loop geometry', Nuclear Phys. B 288(3-4) (1987), 578-588.

[11] V. Mathai and D. Quillen, 'Superconnections, Thom classes, and equivariant differential forms', Topology 25(1) (1986), 85-110.

[12] M. K. Murray and D. Stevenson, 'Higgs fields, bundle gerbes and string structures', Comm. Math. Phys. 243(3) (2003), 541-555.

[13] M. K. Murray and R. F. Vozzo, 'The caloron correspondence and higher string classes for loop groups', J. Geom. Phys. 60(9) (2010), 1235-1250.

[14] M. K. Murray and R. F. Vozzo, 'Circle actions, central extensions and string structures', Int. J. Geom. Methods Mod. Phys. 7(6) (2010), 1065-1092.

[15] M. S. Narasimhan and S. Ramanan, 'Existence of universal connections', Amer. J. Math. 83 (1961), 563-572.

[16] M. S. Narasimhan and S. Ramanan, 'Existence of universal connections. II', Amer. J. Math. 85 (1963), 223-231.

[17] A. Pressley and G. Segal, Loop Groups, Oxford Mathematical Monographs (Oxford University Press, New York, 1986). 
[18] R. Schlafly, 'Universal connections', Invent. Math. 59(1) (1980), 59-65.

[19] R. F. Vozzo, 'Loop groups, Higgs fields and generalised string classes', PhD Thesis, School of Mathematical Sciences, University of Adelaide, 2009.

RAYMOND F. VOZZO, School of Mathematical Sciences, University of Adelaide, Adelaide SA 5005, Australia

e-mail: raymond.vozzo@adelaide.edu.au 